\title{
Modular pathway engineering for the microbial production of branched-chain fatty alcohols
}

Wen Jiang ${ }^{1}$, James B. Qiao' ${ }^{1}$ Gayle J. Bentley ${ }^{2,4}$, Di Liu' ${ }^{1}$ and Fuzhong Zhang ${ }^{1,2,3^{*}}$ (])

\begin{abstract}
The intrinsic structural properties of branched long-chain fatty alcohols (BLFLs) in the range of C12 to C18 make them more suitable as diesel fuel replacements and for other industrial applications than their straight-chain counterparts. While microbial production of straight long-chain fatty alcohols has been achieved, biosynthesis of BLFLs has never been reported. In this work, we engineered four different biosynthetic pathways in Escherichia coli to produce BLFLs. We then employed a modular engineering approach to optimize the supply of a-keto acid precursors and produced either odd-chain or even-chain BLFLs with high selectivity, reaching 70 and $75 \%$ of total fatty alcohols, respectively. The acyl-ACP and alcohol-producing modules were also extensively optimized to balance enzyme expression level and ratio, resulting in a 6.5 -fold improvement in BLFL titers. The best performing strain overexpressed 14 genes from 6 engineered operons and produced $350 \mathrm{mg} / \mathrm{L}$ of BLFLs in fed-batch fermenter. The modular engineering strategy successfully facilitated microbial production of BLFLs and allowed us to quickly optimize new BLFL pathway with high titers and product specificity. More generally, this work provides pathways and knowledge for the production of BLFLS and BLFL-related, industry-relevant chemicals in high titers and yields.
\end{abstract}

Keywords: Branched long-chain fatty alcohols, Branched-chain fatty acids, Advanced biofuels, Modular pathway engineering

\section{Background}

Finite energy resources and increased environmental concerns demand the development of sustainable and renewable approaches to the production of fuels, chemicals, and materials. Engineering microbial metabolic pathways to synthesize desired products is an attractive approach. With recent advances in genetic techniques and metabolic engineering methodologies, various engineered microbes have been developed to produce an array of chemicals derived from inexpensive and renewable substrates $[1,2]$. Target molecules are typically designed to replace or mimic those obtained from petroleum or other non-renewable sources [3, 4]. Among these

\footnotetext{
*Correspondence: fzhang@seas.wustl.edu

1 Department of Energy, Environmental and Chemical Engineering,

Washington University in St. Louis, 1 Brookings Drive, Campus Box 1180,

Saint Louis, MO 63130, USA

Full list of author information is available at the end of the article
}

chemicals, long-chain fatty alcohols (LCFLs) in the range of $\mathrm{C} 12-\mathrm{C} 18$ have numerous applications as fuels, emollients, plasticizers, thickeners, and detergents [5-7]. At the industrial scale, LCFLs are currently produced either through hydrogenation of pretreated natural fats and oils (oleochemicals) or hydroformylation of petrochemicals (e.g. crude oil, natural gas) [8-10]. Because both processes require harsh reaction conditions and release harmful byproducts to the environment [11], microbial production of fatty alcohols from renewable sugars is a promising alternative.

Long-chain fatty alcohols have been recently biosynthesized from engineered microbes through fatty acid biosynthetic pathway [5, 10-15]. The majority of previously biosynthesized LCFLs contain straight aliphatic chains. Straight LCFLs have relatively high freezing points and viscosities compared to their branched-chain isomers, thus limiting their low-temperature operability-an 
essential feature for industrial emollients, lubricants, detergents, and most especially, diesel fuels [16, 17]. For example, the most abundant biosynthetically produced LCFL, straight-chain palmityl alcohol (C16, often called cetyl alcohol), melts at $49.3{ }^{\circ} \mathrm{C}$ and exists as a solid at room temperature, making it difficult to use as a lubricant or an aircraft hydraulic fluid [18]. In comparison, the branched isomer, isocetyl alcohol, is a clear liquid at room temperature (melting point $-30{ }^{\circ} \mathrm{C}$ ) with significantly enhanced fluidity and low-temperature operability, and thus is used in a number of detergent formulations that require low-temperature operability [19]. Introducing degrees of unsaturation has been used as an alternative method to improve the fluidity of straight-chain molecules [20]. However, these molecules are vulnerable to oxidation and color instability, both of which do not occur in BLFLs, even at high temperatures and pressures [17]. BLFLs additionally are ranked low in eye and skin irritation, allowing their use in cosmetics and fragrances [16]. Given the clear breadth BLFL applications, chemical engineers have developed methods to synthesize naturally scarce BLFLs [21-23]. BLFLs are currently produced from straight LCFLs using a multi-step, catalytic process that requires high temperature $\left(180-300{ }^{\circ} \mathrm{C}\right)$, strong acids/bases, and multiple operation unites, and the conversion process is unpredictable [16]. Recently, branched-short-chain alcohols in the range of $(\mathrm{C} 3-\mathrm{C} 7)$ were produced by engineered microbes $[24,25]$ as gasoline replacements. However, due to their short chainlength and high volatility, branched-short-chain alcohols are not suitable for the above-mentioned applications. Given BLFLs' lack of natural abundance, their complex synthesis process, and the demand for their distinctive properties, it is imperative to develop biosynthetic approaches to produce BLFLs from renewable feedstock.

Since straight LCFLs have been biologically or chemically derived from free fatty acid precursors [26], BLFLs theoretically can be derived from branched-chain fatty acids (BCFAs). BCFAs have been recently biosynthesized in engineered $E$. coli by overexpressing a heterologous branched-chain $\alpha$-keto acid dehydrogenase complex (BKD), a branched-chain-acyl-CoA-specific $\beta$-ketoacylacyl-carrier protein $(\mathrm{FabH})$ and a thioesterase (TesA) $[27,28]$. This system ultimately produced BCFA in high percentages from glucose $(181 \mathrm{mg} / \mathrm{L}$ and $80 \%$ BCFA) by engineering a protein lipoylation pathway and an $\alpha$-keto acid biosynthetic pathway. However, highly efficient conversion of BCFAs to BLFLs has not been explored. It was not known whether the branched-chain intermediates, such as branched long-chain acyl-ACPs, BCFAs, and branched long-chain acyl-CoAs, are compatible with alcohol-conversion enzymes, even though some enzymes have activities towards branched short-chain-ACP or
CoA intermediates $[29,30]$. Furthermore, because the BLFL pathway involves in multiple untested reaction, balancing activities of each sub-pathway to prevent accumulation of branched-chain intermediate is necessary to obtain high titer and yield [31-34].

To achieve these goals, we constructed and tested four metabolic pathways that convert branched-chain acylACPs to BLFLs. To optimize productivity, the entire BLFL pathway was divided into three modules (Fig. 1): an $\alpha$-keto acid synthesis module that converts glucose to $\alpha$-keto acids, an acyl-ACP generation module that converts $\alpha$-keto acids to branched-chain acyl-ACPs, and an alcohol formation module that converts branched-chain acyl-ACPs to final branched-chain products. To begin, we experimentally determined the most efficient enzyme combinations to convert branched-chain acyl-ACPs to BLFLs using an engineered branched-chain-acyl-ACPproducing basal strain (BC33). This initial pathway identification allowed us to ascertain whether BLFL production was even feasible, given the available enzymes. Next, we optimized each module separately and later the entire pathway collectively to produce even-chain-iso and odd-chain-iso fatty alcohols from glucose. Our modular approach enabled us to isolate each module for testing and optimization, without confounding the system by simultaneously engineering the other modules.

\section{Methods}

\section{Materials and media}

Phusion DNA polymerase was purchased from New England Biolabs (Beverly, MA, USA). Restriction enzymes, T4 ligase, gel purification kits, and plasmid miniprep kits were purchased from Thermo Fisher Scientific (Waltham, Massachusetts, USA). All primers were synthesized by Integrated DNA Technologies (Coralville, IA, USA). BCFA standards (Bacterial Acid Methyl Ester Mix), SCFA standards (GLC-20 and GLC-30), and all the other reagents were purchased from Sigma Aldrich (St. Louis, MO, USA).

Minimal medium (M9 medium supplemented with $75 \mathrm{mM}$ MOPS at pH 7.4, $2 \mathrm{mM} \mathrm{MgSO}_{4}, 1 \mathrm{mg} / \mathrm{L}$ thiamine, $50 \mu \mathrm{g} / \mathrm{mL}$ lipoic acid, $10 \mu \mathrm{M} \mathrm{FeSO}_{4}, 0.1 \mathrm{mM} \mathrm{CaCl}_{2}$, and micronutrients, including $3 \mu \mathrm{M}\left(\mathrm{NH}_{4}\right)_{6} \mathrm{Mo}_{7} \mathrm{O}_{24}, 0.4 \mathrm{mM}$ boric acid, $30 \mu \mathrm{M} \mathrm{CoCl}_{2}, 15 \mu \mathrm{M} \mathrm{CuSO}_{4}, 80 \mu \mathrm{M} \mathrm{MnCl}_{2}$, and $10 \mu \mathrm{M} \mathrm{ZnSO}_{4}$ ) containing $2 \%$ glucose and $0.5 \%$ yeast extract as carbon sources was used for cell growth and fatty acid production.

\section{Plasmids and strains}

Plasmids and oligonucleotides used in this study are listed in Table 1. Genes encoding S. elongatus AAR (aar), M. marinum CAR (car), and B. subtilis Sfp (sfp) were codon-optimized for $E$. coli expression and synthesized 


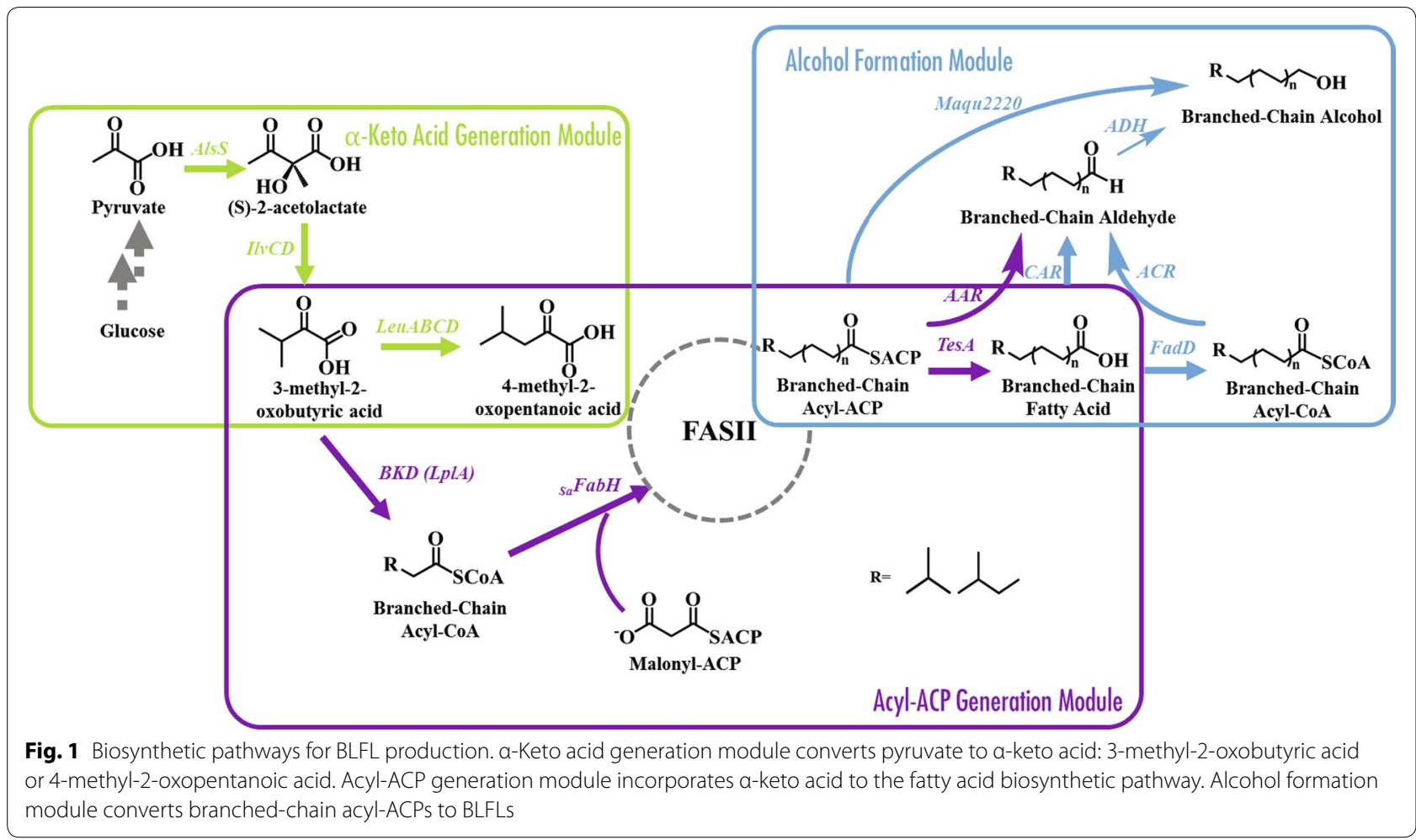

by Integrated DNA Technologies (Coralville, IA, USA). Genes encoding ACR (maqu2507) and Maqu2220 (maqu2220) were amplified by PCR from templates provided by Dr. Brett M. Barney (University of Minnesota, $\mathrm{MN}$ ) and Dr. Jay D. Keasling (Joint BioEnergy Institute, CA). To create all plasmids (Table 1), the corresponding genes were assembled into the backbones of BioBrick plasmids [35], using either restriction sites or GoldenGate DNA assembly method [36].

Escherichia coli DH10B was used for cloning purposes. E. coli strain CL111 [37] (a gift from Dr. Cronan's Lab, University of Illinois at Urbana-Champaign) was used for production purposes. Strain BC30 was created by integrating the $b k d$ operon (lpdV, bkdAA, bkdAB, and $b k d B)$ at the $f a d E$ locus in the genome under the control of a $\mathrm{P}_{\text {LacUv5 }}$ promoter, using a previously described technique [38]. Plasmid pSa-P ecfabH $^{-} \mathrm{Sa}^{\mathrm{fabH}}$ was transformed into BC30 strain, and the ${ }_{S e} f a b H$ was knocked out by P1 transduction, creating strain BC33 [37]. Strains BO33A$J$ were created by transforming the corresponding plasmids (Table 2) into BC33 competent cells, respectively. Strain $\mathrm{BC} 43$ was created by switching native leuABCD promoter $\mathrm{P}_{\text {leuLp }}$ and $\mathrm{P}_{\text {leuLp2 }}$ to $\mathrm{P}_{\text {lacuv5 }}$ promoter, using a previously described CRISPR-Cas9 gene replacement method [39]. Similarly, to create strain BC63, the fadRlplA operon under the control of a $\mathrm{P}_{\mathrm{LacUv} 5}$ promoter was integrated to the $l d h A$ locus of $\mathrm{BC} 43$ strain by the same
CRISPR-Cas9 gene replacement method. Strains BO63V and BO63L were created by transforming the corresponding plasmids into strain BC63.

\section{Cell culturing and a-keto acids supplementation}

Cells were pre-cultivated in LB medium with proper antibiotics. Overnight cultures were inoculated $2 \% \mathrm{v} / \mathrm{v}$ into M9 minimal medium (described in "Materials and media" section) with corresponding antibiotics for adaptation. Overnight cultures in minimal medium were then used to inoculate $5 \mathrm{~mL}$ of the same fresh minimal medium, with an initial $\mathrm{OD}_{600}$ of 0.08 . When $\mathrm{OD}_{600}$ reached 0.8 , cells were induced with proper inducers $(1 \mathrm{mM}$ isopropyl $\beta$-D-1-thiogalactopyranoside (IPTG), 0.4\% arabinose and/or $200 \mathrm{nM}$ anhydrotetracycline (aTc), or otherwise specified). For $\alpha$-keto acid supplementation experiments, one of the $\alpha$-keto acids (3-methyl-2-oxobutyric acid, 3-methyl-2-oxopentanoic acid, or 4-methyl-2-oxopentanoic acid) was added at $\mathrm{OD}_{600}=0.8$ to a final concentration of $1 \mathrm{~g} / \mathrm{L}$. Cells were harvested 3 days after induction.

\section{Fermentation}

Fed-batch fermentation was carried out using a New Brunswick Bioflo 110 fermenter with a pH meter, a dissolved oxygen electrode, and a temperature electrode. M9 medium (described in "Materials and media" section, $500 \mathrm{~mL}$ ) was inoculated with an overnight culture 
Table 1 Plasmids used in this research

\begin{tabular}{|c|c|c|c|c|}
\hline Plasmids & Replication ori & Overexpressed operon & Resistance & References \\
\hline pSa-P ecfabH $^{-}{ }{ }_{a} f a b H$ & SC101 & $P_{\text {ecfabH }}{ }^{-}{ }_{a} f a b H$ (S. aureus) & $A m p^{R}$ & {$[28]$} \\
\hline pB5k-aar-Ip/A & pBBR1 & 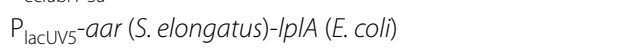 & $\operatorname{Kan}^{R}$ & This study \\
\hline pB5k-sfp-car & pBBR1 & $P_{\text {lacuv5-sfp (B. subtilis)-car (M. marinum) }}$ & $\operatorname{Kan}^{R}$ & This study \\
\hline pE8c-tesA-Ip/A & ColE1 & $P_{B A D}$-tesA (E. coli)-Ip/A (E. coli) & $\mathrm{Cm}^{\mathrm{R}}$ & This study \\
\hline pB5k-maqu2507-Ip/A & pBBR1 & 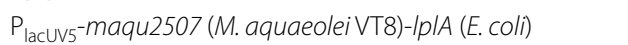 & $\operatorname{Kan}^{R}$ & This study \\
\hline pE5c-tesA-fadD & ColE1 & $\mathrm{P}_{\text {lacuv5 }}$-tesA (E. coli)-fadD (E. coli) & $\mathrm{Cm}^{\mathrm{R}}$ & [48] \\
\hline pB5k-maqu2220 & pBBR1 & $\mathrm{P}_{\text {lacuv5-maqu2220 (M. aquaeolei VT8) }}$ & $\operatorname{Kan}^{R}$ & [48] \\
\hline pB5k-maqu2220-Ip/A & pBBR1 & 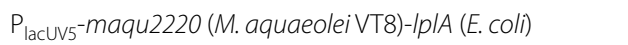 & $\operatorname{Kan}^{\mathrm{R}}$ & This study \\
\hline pB5k-adhA & pBBR1 & $\mathrm{P}_{\text {lacuv5 }}$-adhA (L. lactis) & $\operatorname{Kan}^{R}$ & This study \\
\hline pB5k-yqhD & pBBR1 & $\mathrm{P}_{\text {lacuvs}}$-yqhD (E. coli) & $\operatorname{Kan}^{R}$ & This study \\
\hline pB5k-yjgB & pBBR1 & $\mathrm{P}_{\text {lacuv5}}-y j g B(E . c o l i)$ & $\operatorname{Kan}^{R}$ & This study \\
\hline pA8c-aar-Ip/A & pA15a & $\mathrm{P}_{\mathrm{BAD}}-a \operatorname{ar}($ S. elongatus)-IplA (E. coli) & $\mathrm{Cm}^{\mathrm{R}}$ & This study \\
\hline pB2k-alsS-ilvCD-yjgB & pBBR1 & $P_{\text {tet }}$-alsS (B. subtilis)-ilvCD (E. coli)-yjgB(E. coli) & $\operatorname{Kan}^{R}$ & This study \\
\hline pE2c-aar-Ip/A-yjgB & ColE1 & $P_{\text {tet }}$-aar (S. elongatus)-IplA (E. coli)-yjgB (E. coli) & $\mathrm{Cm}^{\mathrm{R}}$ & This study \\
\hline pA6k-alsS-ilvCD & pA15a & $\mathrm{P}_{\text {Llaco-alsS (B. subtilis)-ilvCD (E. coli) }}$ & $\operatorname{Kan}^{\mathrm{R}}$ & [43] \\
\hline pB2k-alss-ilvCD & pBBR1 & $P_{\text {tet }}$-alsS (B. subtilis)-ilvCD (E. coli) & $\operatorname{Kan}^{\mathrm{R}}$ & This study \\
\hline pB5c-aar-Ip/A-yjgB & pBBR1 & 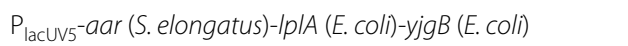 & $\mathrm{Cm}^{\mathrm{R}}$ & This study \\
\hline pB5c-maqu2220-Ip/A & pBBR1 & 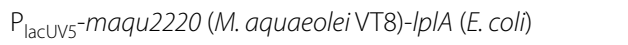 & $\mathrm{Cm}^{\mathrm{R}}$ & This study \\
\hline pA8c-leuA ${ }^{\text {mut }} B C D$ & pA15a & $\mathrm{P}_{\mathrm{BAD}}$-leuA ${ }^{\text {mut }} B C D(E$. coli $)$ & $\mathrm{Cm}^{\mathrm{R}}$ & This study \\
\hline pE8c-leuA ${ }^{\text {mut }} B C D$ & ColE1 & $\mathrm{P}_{\mathrm{BAD}}-l e u A^{\text {mut }} B C D(E$. coli $)$ & $\mathrm{Cm}^{\mathrm{R}}$ & This study \\
\hline pB5k-maqu2220-leuA $A^{\text {mut }} B C D$ & pBBR1 & 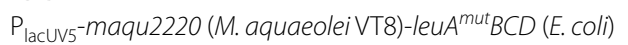 & $\operatorname{Kan}^{R}$ & This study \\
\hline pA8c-Ip/A & pA15a & $\mathrm{P}_{\mathrm{BAD}}-\mid p / A(E . c o l i)$ & $\mathrm{Cm}^{\mathrm{R}}$ & This study \\
\hline pE8c-adhA & ColE1 & $\mathrm{P}_{\mathrm{BAD}}-a d h A$ (L. lactis) & $\mathrm{Cm}^{\mathrm{R}}$ & This study \\
\hline pE8c-yqhD & ColE1 & $\mathrm{P}_{\mathrm{BAD}}-y q h D(E$. coli $)$ & $\mathrm{Cm}^{\mathrm{R}}$ & This study \\
\hline pE8c-yjgB & ColE1 & $\mathrm{P}_{\mathrm{BAD}}-\mathrm{yjg} \mathrm{B}$ (E. coli) & $\mathrm{Cm}^{\mathrm{R}}$ & This study \\
\hline pE2s-alsS-ilvCD & ColE1 & $P_{\text {tet }}-$ alsS (B. subtilis)-ilvCD (E. coli) & $\operatorname{Spec}^{R}$ & This study \\
\hline
\end{tabular}

of strain BO63L to an initial $\mathrm{OD}_{600}$ to 0.08 , along with appropriate antibiotics and $0.001 \%$ Antifoam 204. The fermentation was initiated with the following settings: Temperature was set to $30^{\circ} \mathrm{C}, \mathrm{pH}$ was controlled at 7.4 by automatic feeding of $6 \mathrm{~N}$ ammonium hydroxide, the airflow rate was kept at $1.5 \mathrm{~L} / \mathrm{min}$, and the average stirring rate was maintained at $500 \mathrm{rpm}$. Gene expression was induced at $\mathrm{OD}_{600}=10$ by addition of $1 \mathrm{mM}$ IPTG, $0.4 \%$ arabinose, and $0.22 \mu \mathrm{M}$ aTc (final concentration). A glucose stock solution (400 g/L glucose and $12 \mathrm{~g} / \mathrm{L} \mathrm{MgSO}_{4}$ ) was intermittently pulsed into the bioreactor to re-supply glucose, and a yeast extract solution (20\%) was intermittently added into the bioreactor. Broth samples $(\sim 3 \mathrm{~mL})$ were collected at a series of time points to measure cell density and alcohol titer.

\section{Quantification of the fatty alcohols}

For the quantification of alcohols, $1 \mathrm{~mL}$ of cell culture was acidified with $100 \mu \mathrm{L}$ of concentrated $\mathrm{HCl}(12 \mathrm{~N})$. Alcohols were extracted twice with $0.5 \mathrm{~mL}$ ethyl acetate, and the organic layers were isolated. Next, $200 \mu \mathrm{L}$ of the organic layer from each sample was transferred to one $2 \mathrm{~mL}$ clear glass GC vial (Agilent Technologies, Santa Clara, CA), mixed with $200 \mu \mathrm{L}$ of $N, O$-bis(trimethylsilyl) trifluoroacetamide (BSTFA) with $1 \% \mathrm{v} / \mathrm{v}$ chlorotrimethylsilane, and incubated at $60{ }^{\circ} \mathrm{C}$ for $2 \mathrm{~h}$. Alcohol derivatives were quantified using a GC-MS (Hewlett-Packard model 7890 A, Agilent Technologies) equipped with a $30 \mathrm{~m}$ DB5-MS column (J\&W Scientific) and a mass spectrometer (5975C, Agilent Technologies) or a FID (Agilent Technologies) detector. For each sample, the column was equilibrated at $80{ }^{\circ} \mathrm{C}$, followed by a ramp to $300{ }^{\circ} \mathrm{C}$ at $20{ }^{\circ} \mathrm{C} / \mathrm{min}$, and was then held at $300{ }^{\circ} \mathrm{C}$ for $3 \mathrm{~min}$. Individual alcohol peaks were identified by comparing their retention time to that of a standard (a mixture of 1-tetradecanol, 1-hexadecanol, and 1-octadecanol, prepared and derivatized identically to samples) and by comparing their mass spectra to the National Institute of Standards and Technology (NIST) Mass Spectral Library. Concentrations of each alcohol were determined by comparing the area of each sample peak to a standard curve generated by standards eluted using the same method. Product 
Table 2 Strains used in this research

\begin{tabular}{|c|c|c|}
\hline Strains & Relevant genotype & References \\
\hline \multicolumn{3}{|l|}{ Parental strains } \\
\hline CL111 & UB1005, attHK022::(plsX'fabH; aadA) fabH::kan & [37] \\
\hline CL111(Akan) & UB1005, attHK022::(p/sX'fabH; aadA), $\Delta$ kan & [28] \\
\hline BC30 & $C L 111(\Delta k a n)$ fadE:: $b_{s} \mid p d V_{b s} b k d A A_{b s} b k d A B-{ }_{b s} b k d B$ & This study \\
\hline BC33 & 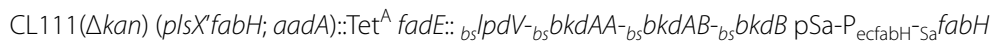 & This study \\
\hline $\mathrm{BC} 43$ & BC33 leuO-leuL-leuA.:P lacuvs-leuA $A^{\text {mut }}$ & This study \\
\hline BC63 & BC43 IdhA::fadR-Ip/A & This study \\
\hline \multicolumn{3}{|c|}{ Alcohol-producing strains } \\
\hline $\mathrm{BO} 33 \mathrm{~A}$ & BC33 pB5k-aar-Ip/A & This study \\
\hline BO33B & BC33 pB5k-sfp-car, pE8c-tesA-Ip/A & This study \\
\hline $\mathrm{BO} 33 \mathrm{C}$ & BC33 pB5k-maqu2507-Ip/A, pE5c-tesA-fadD & This study \\
\hline BO33D & BC33 pB5k-maqu2220-Ip/A & This study \\
\hline BO33E1 & BC33 pA8c-aar-Ip/A, pB5k-adhA & This study \\
\hline BO33E2 & BC33 pA8c-aar-Ip/A, pB5k-yqhD & This study \\
\hline BO33E3 & BC33 pA8c-aar-Ip/A, pB5k-yjgB & This study \\
\hline BO33E4 & BC33 pE2c-aar-Ip/A-yjgB & This study \\
\hline $\mathrm{BO} 33 \mathrm{~F}$ & BC33 pA8c-aar-Ip/A, pB2k-alsS-ilvCD-yjgB & This study \\
\hline BO33F1 & BC33, pE2c-aar-Ip/A-yjgB, pA6k-alsS-ilvCD & This study \\
\hline BO33F2 & BC33, pE2c-aar-Ip/A-yjgB, pB2k-alsS-ilvCD & This study \\
\hline $\mathrm{BO} 33 \mathrm{~F} 3$ & BC33, pB5c-aar-Ip/A-yjgB, pA6k-alsS-ilvCD & This study \\
\hline BO33G1 & BC33, pA8c-leuA ${ }^{\text {mut }} B C D$, pB5k-aar-lp/A & This study \\
\hline $\mathrm{BO} 33 \mathrm{G} 2$ & BC33, pE8c-leuA ${ }^{\text {mut }} B C D$, pB5k-aar-Ip/A & This study \\
\hline $\mathrm{BO} 33 \mathrm{H} 1$ & BC33 pB5k-maqu2220-Ip/A, pE8c-adhA & This study \\
\hline $\mathrm{BO} 33 \mathrm{H} 2$ & BC33 pB5k-maqu2220-Ip/A, pE8c-yqhD & This study \\
\hline $\mathrm{BO} 33 \mathrm{H} 3$ & BC33 pB5k-maqu2220-Ip/A, pE8c-yjgB & This study \\
\hline BO3311 & BC33, pA6k-alsS-ilvCD, pB5c-maqu2220-Ip/A & This study \\
\hline BO3312 & BC33, pE2k-alsS-ilvCD, pB5c-maqu2220-Ip/A & This study \\
\hline BO33」1 & BC33, pA8c-leuA ${ }^{\text {mut }} B C D$, pB5k-maqu2220-Ip/A & This study \\
\hline BO33J2 & BC33, pA8c-Ip/A, pB5k-maqu2220-leuA ${ }^{\text {mut }} B C D$ & This study \\
\hline BO431 & BC43 pB5c-maqu2220-Ip/A, pE2s-alsS-ilvCD & This study \\
\hline BO63V & BC63 pB5k-maqu2220, pE2s-alsS-ilvCD & This study \\
\hline BO63L & BC63 pB5k-maqu2220, pE2s-alsS-ilvCD, pA8c-leuABCD & This study \\
\hline
\end{tabular}

titer for each strain was measured in biological triplicate (starting from three different colonies) and average values are reported.

\section{Results}

Engineering alcohol formation modules in BCFA-producing strains

To create the branched-chain-acyl-ACP-producing basal strain $\mathrm{BC} 33$, the E. coli $f a b H$ was first replaced by Staphylococcus aureus fabH ( $\left.{ }_{S a} f a b H\right)$, a modification which was previously demonstrated to enhance branched-chain fatty acid production [28]. The acyl-CoA dehydrogenase gene $(f a d E)$ was next replaced by Bacillus subtilus $b k d$, functionally inhibiting $\beta$-oxidation $(\Delta f a d E)$ and allowing the activation of branched-chain $\alpha$-keto acids to branched-chain acyl-CoAs that can enter the FASII system $(f a d E:: b k d)$ (Tables 1,2$)$. Next, three alcohol formation modules were separately constructed in strain BC33 (Table 2), resulting in alcohol-producing strains BO33A-C. Strain BO33A utilizes the Synechococcus elongatus acyl-ACP reductase (AAR, encoded by aar, Fig. 1) to convert acyl-ACPs to fatty aldehydes [13]. Strain BO33B first generates FFAs via expression of the E. coli cytosolic thioesterase (TesA), which are then converted to fatty aldehydes by the Mycobacterium marinum carboxylic acid reductase (CAR, encoded by car) with Bacillus subtilis Sfp (encoded by $s f p$ ) coexpression [5] (Fig. 1). Strain BO33C also expresses cytosolic TesA to generate FFAs and overexpresses an E. coli acyl-CoA synthetase (FadD) to activate FFAs to fatty acyl-CoAs, which 
are then converted to aldehydes by overexpressing an acyl-CoA reductase from Marinobacter aquaeolei VT8 (ACR, encoded by maqu2507) [40] (Fig. 1). All strains rely on native $E$. coli alcohol dehydrogenases (ADHs) to reduce aldehydes to alcohols. The native $E$. coli lipoyl ligase (encoded by $l p l A$ ) is also expressed in all strains to improve the lipoylation of 2-oxoacid dehydrogenases including $\mathrm{BKD}$, which requires lipoylation to function [27].

We first sought to test the three alcohol formation modules by assessing their capacities to convert branched-chain acyl-ACPs to BLFLs. Strains BO33A-C were cultivated at previously determined optimal temperatures for each pathway (see "Methods" section) [5, $12,41]$ and supplemented with $1 \mathrm{~g} / \mathrm{L}$ of 4-methyl-2-oxopentanoic acid, the most favorable $\alpha$-keto acids for BCFA production [28]. Each strain produced some BLFLs, representing the first report of BLFL production in $E$. coli. The CAR pathway (BO33B) and the ACR pathway (BO33C) produced 28 and $18 \mathrm{mg} / \mathrm{L}$ BLFLs, respectively. In addition, the chain lengths of the products range from C14 to C18, consistent with the FFA profile of the TesAoverexpressing strain [42]. Meanwhile, the AAR pathway (BO33A) produced the highest BLFL titer among these three strains: $54 \mathrm{mg} / \mathrm{L}$, comprising $84 \%$ of the total fatty alcohols (Fig. 2a). The branch and chain-length profile of BLFLs produced in BO33A is consistent with the profile of BCFAs produced by strains with similar genetic backgrounds as previously reported (Fig. 2b) [27], indicating that the AAR pathway has little preference between straight-chain and branched-chain acyl-ACP substrates. Interestingly, the CAR pathway produced twofold more straight-chain fatty alcohols but sixfold less BLFLs than the AAR pathway, indicating that the CAR pathway is primarily straight-chain-specific. While these results serve as a proof-of-principle that BLFL can be produced at high percentages in $E$. coli, the initial low titers indicate that additional engineering efforts are needed to obtain desirable titers and yields of branched-chain products.

All of the above strains relied upon E. coli native ADHs to convert fatty aldehydes to alcohols. We next sought to increase BLFL production in strain BO33A by overexpression of ADHs. Three unique ADHs, adhA from Lactococcus lactis [43], and $y q h D$ and $y j g B$ [5, 43, 44] from E. coli, were overexpressed in strain BO33A, resulting in strains BO33E1, BO33E2, and BO33E3, respectively. When cultivated under the same conditions and with $1 \mathrm{~g} / \mathrm{L}$ 4-methyl-2-oxopentanoic acid, strains BO33E1 and
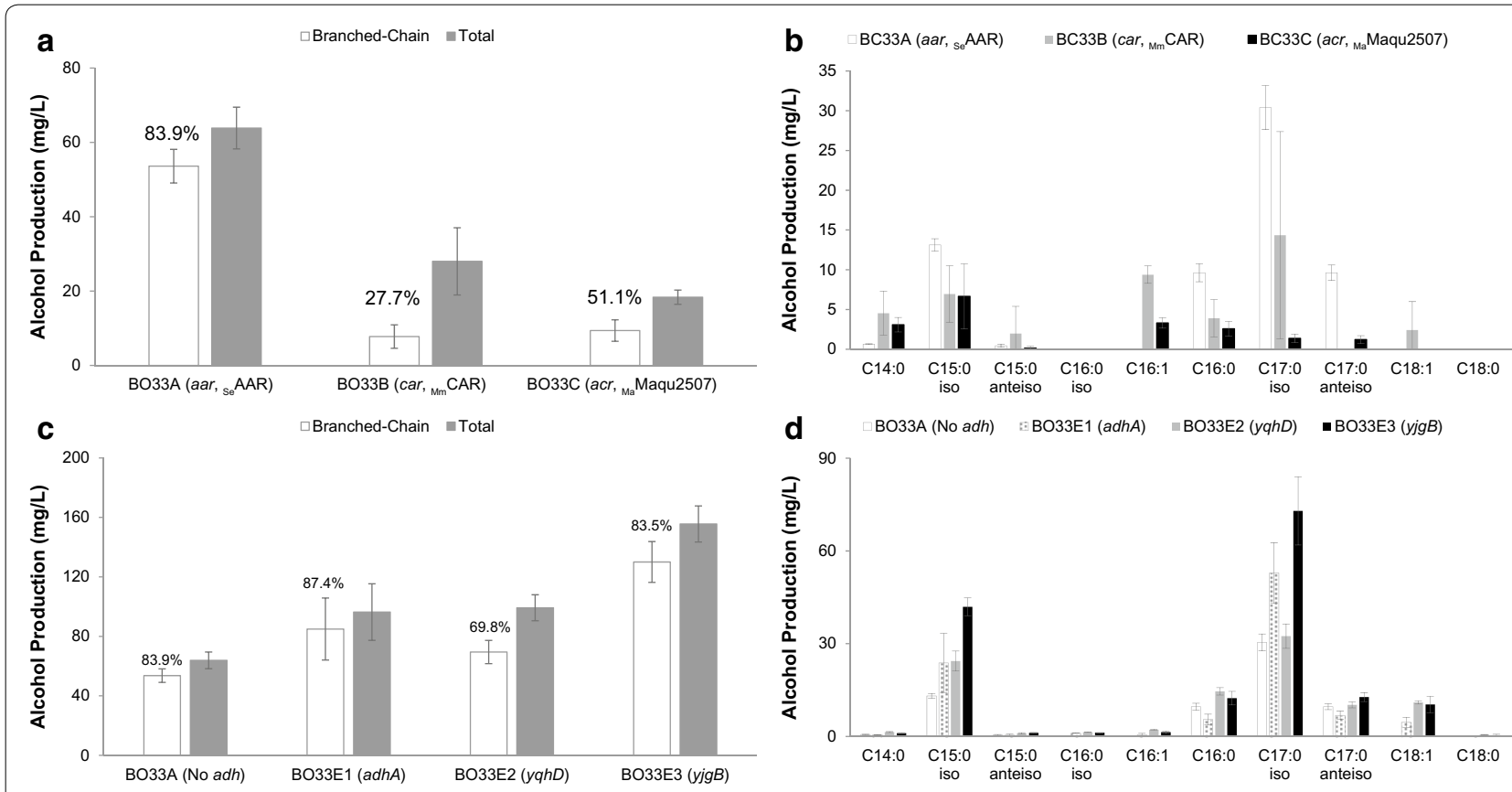

Fig. 2 Testing three alcohol formation modules. a Overall titer distribution and $\mathbf{b}$ chain-length of three BLFL-producing strains with different

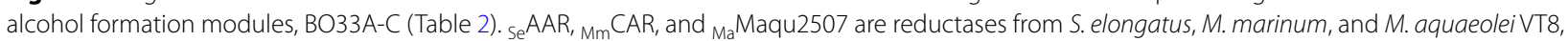
respectively. $\mathbf{c}, \mathbf{d}$ Effect of alcohol dehydrogenase overexpression on BLFL production. Alcohol profiles of strain BO33A (without adh overexpression) are compared with those from strain BO33E1 (expressing adhA from L. lactis), BO33E2 (expressing yqhD from E. coli), and BO33E3 (expressing yjgB from E. coli). All cultures were supplemented with $1 \mathrm{~g} / \mathrm{L}$ 4-methyl-2-oxopentanoic acid. Cells were cultivated and induced as described in "Methods" section 
BO33E2 produced 85 and $69 \mathrm{mg} / \mathrm{L}$ BLFLs, respectively (Fig. 2c, d). Strain BO33E3 produced $130 \mathrm{mg} / \mathrm{L}$ BLFLs, comprising $83 \%$ of total alcohols, a 2.4 -fold enhancement over BO33A. Specifically, $73 \mathrm{mg} / \mathrm{L}$ of 15 -methylhexadecanol and $42 \mathrm{mg} / \mathrm{L}$ of 13-methyltetradecanol were produced (Fig. 2c), converting $8 \%$ of the supplemented 4-methyl-2-oxopentanoic acid and making odd-chainiso alcohols the major $\mathrm{BC}$ alcohol species. Both BO33E1 and BO33E3 had similar fatty alcohol production profiles to that of strain BO33A, indicating that AdhA and YjgB did not selectively determine chain length or structure. In addition, no aldehyde was detected in BO33E3 culture, indicating the conversion of aldehyde to alcohol is complete.

\section{Engineering 3-methyl-2-oxobutyric acid generation module to produce even-chain-iso fatty alcohols from glucose}

Modular pathway assembly can facilitate step-wise optimizations. With the downstream alcohol formation module optimized, we next sought to improve the production of BLFL from glucose by engineering and optimizing the $\alpha$-keto acid generation module. In this module, we built upon the downstream-optimized strain BO33E3 to add the capacity for even-chain-iso fatty alcohol production from glucose via the precursor 3-methyl-2-oxobutyric acid (Fig. 3a).

Since the $B$. subtilis alsS and the E. coli ilvCD have been used to accumulate intracellular 3-methyl-2-oxobutyric acid, we first cloned them with $y j g B$ under the control of a strong $\mathrm{P}_{\text {tet }}$ promoter in a medium-copy-number BBR1 origin plasmid (Table 1). The resulting plasmid, pB2kalsS-ilvCD-yjgB, was co-transformed with pA8c-aar-lplA (aar and $l p l A$ under the control of a $\mathrm{P}_{\mathrm{BAD}}$ promoter in a pA15a origin plasmid) into strain BC33. Cultivation of the resulting strain $\mathrm{BO} 33 \mathrm{~F}$ in the absence of $\alpha$-keto acid supplementation produced $34 \mathrm{mg} / \mathrm{L}$ of BLFLs, comprising $44 \%$ of the total alcohols (Fig. 3b; Additional file 1: Figure S1). Because the composition of the $\alpha$-keto acid determines the final chain structure, the final BLFL composition should mirror the cellular pool of $\alpha$-keto acids. The titer of even-chain-iso BLFLs produced by strain $\mathrm{BO} 33 \mathrm{~F}$ is $14 \mathrm{mg} / \mathrm{L}$, lower than the $25 \mathrm{mg} / \mathrm{L}$ produced by strain BO33E3 when supplemented with 3-methyl2-oxobutyric acid. However, BO33F produced more odd-chain-iso BLFLs, which are also originated from 3-methyl-2-oxobutyric acid via 4-methyl-2-oxopentanoic acid. Thus the total BLFL production of these two strains is comparable, indicating that the 3-methyl-2-oxobutyric acid generation module was effective, although higher expression level of this module might generate more even-chain-iso products.
Optimizing 4-methyl-2-oxopentanoic acid generation module to produce odd-chain-iso fatty alcohols In $E$. coli, the leuABCD operon converts 3-methyl-2-oxobutyric acid to 4-methyl-2-oxopentanoic acid, which is the precursor for odd-chain-iso BLFL biosynthesis. Previous work demonstrated that the ribosome binding sequence (RBS) of native leuA results in weak translation initiation, and $l e u A$ is heavily negatively auto-regulated by free leucine synthesized from 4-methyl-2-oxopentanoic acid [45, 46] (Fig. 4a). In this study, we engineered a mutant operon leu $A^{m u t} B C D$ that contained a feedback-resistant leuA [46, 47] and a strong synthetic RBS (TTTAAGAAGGAGATATACAT). The leuA ${ }^{\text {mut }} B C D$ was cloned in either a low (pA15a) or a high (pColE1) copy number plasmid to create two engineered strains, BO33G1 and BO33G2, respectively. To test the conversion efficiency of the leu $A^{\text {mut }} B C D$ operon, both strains were supplemented with 3-methyl-2-oxobutyric acid (the substrate for LeuABCD, Fig. 4a) during cultivation. Fully functional leu $A^{m u t} B C D$ would result in odd-chainiso BLFL compositions in a similar ratio to our best-performing strain (strain BO33A) when supplemented with 4-methyl-2-oxopentanoic acid. Strain BO33G1 produced $50 \mathrm{mg} / \mathrm{L}$ total BLFL and $47 \mathrm{mg} / \mathrm{L}$ odd-chain-iso fatty alcohol, statistically indistinguishable from the BLFL titer of strain BO33A supplemented with 4-methyl-2-oxopentanoic acid (Fig. 4b; Additional file 1: Figure S2). Strain BO33G1 generated $94 \%$ odd-chain-iso fatty alcohol, indicating complete conversion of 3-methyl-2-oxobutyric acid by the modified LeuA ${ }^{\text {mut }} \mathrm{BCD}$. Conversely, when leu $A^{\text {mut }} B C D$ was expressed under the high copy number plasmid (strain BO33G2), the BLFL titers decreased $(p=0.07)$ and had large standard variations (Fig. 4b), even when the inducer concentration was reduced. Furthermore, overexpression of $l e u A^{\text {mut }} B C D$ from the highcopy number plasmid also decreased cell growth rate and cell density (data not shown). These results indicate that expression of the $\operatorname{leu} A^{\mathrm{mut}} B C D$ operon at too high a level affects cell growth and BLFL production, thus needs to be avoided.

\section{Balancing gene expression levels in all three modules for enhanced BLFL production from glucose}

With the $\alpha$-keto acid generation and the alcohol formation modules optimized separately, we next sought to balance the gene expression levels within the completed pathway. We selected the best-performing genetic elements of each individual module to complete the full pathway: the $\alpha$-keto acid generation module, including als $S$ and ilvCD, the acyl-ACP synthesis module, including aar and $l p l A$, and the alcohol formation module, including $y j g B$ (Fig. 3a). To perform this optimization, 


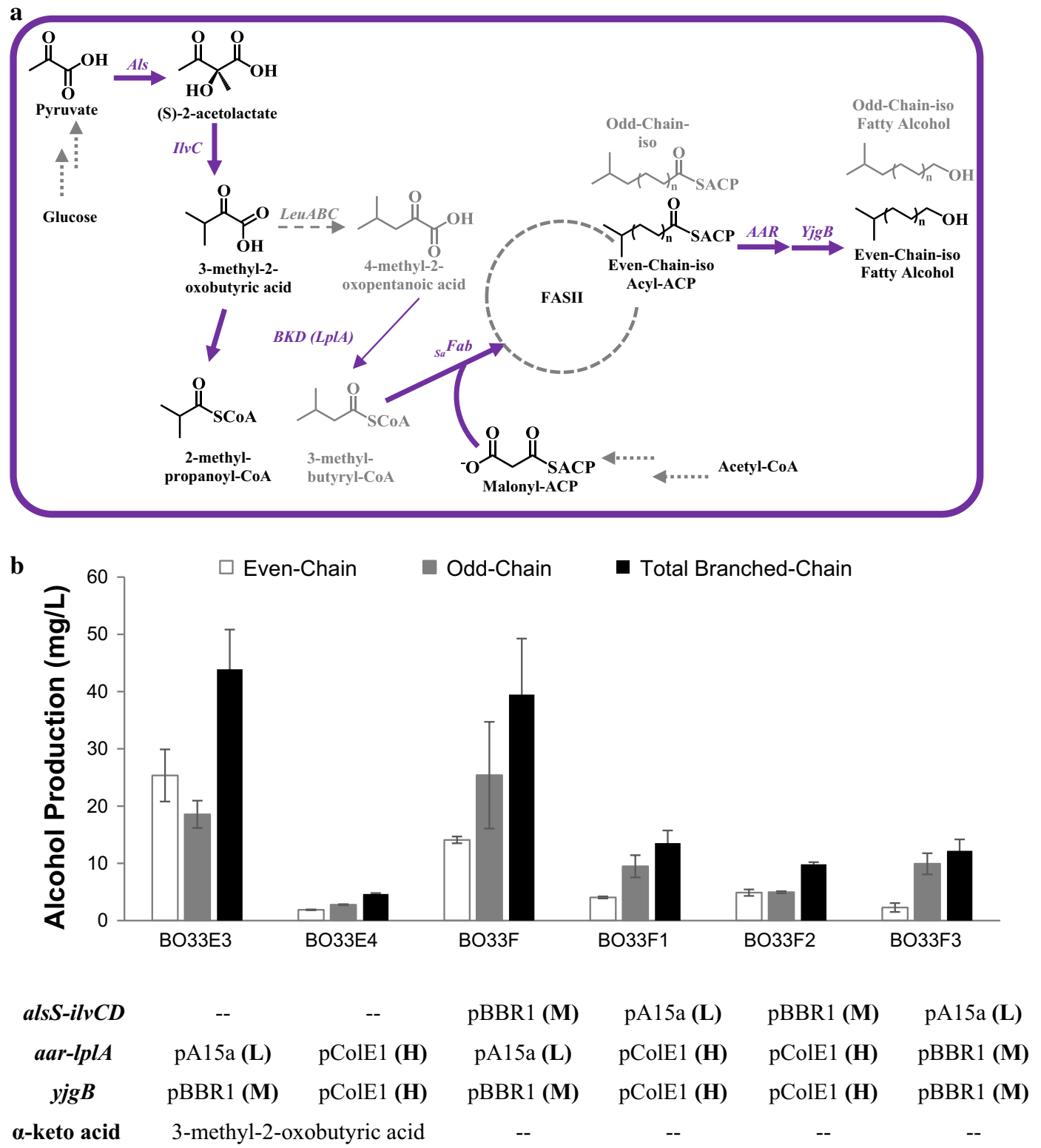

Fig. 3 Balancing three modules to produce even-chain-iso fatty alcohols. Plasmids with different copy numbers and promoters are used to optimize the expression level of the involved pathways. a Biosynthetic pathways for the production of even-chain-iso fatty alcohols. $\mathbf{b}$ Titers of evenchain, odd-chain, and total BLFL of engineered strains. Plasmids with different copy numbers and promoters were employed to bear the involved genes in different strains. The copy number of the plasmids are defined as low (L), medium (M), and high (H). For strain BO33E3-4, $1 \mathrm{~g} / \mathrm{L} 3-\mathrm{methyl-}$ 2-oxobutyric acid was supplemented

we altered gene copy numbers to change the expression level of each module. Compatible plasmids with three different copy numbers were used for this purpose: a high copy number (pColE1, 50 copies per cell), a medium copy number (pBBR1, 17-20 copies per cell), and a low copy number (pA15a, 7-10 copies per cell) [35] (Fig. 3b; Additional file 1: Figure S1). Three additional strains BO33E4,
BO33F1, and BO33F2 were created (Fig. 3b; Additional file 1: Figure S1). All three strains expressed aar, lplA, and $y j g B$ from the high copy number plasmids under the control of a very tight promoter $\left(\mathrm{P}_{\text {tet }}\right)$, but produced less BLFL than strains BO33E3 and BO33F, which expressed aar, lplA, and yjgB from the low or the medium copy number plasmid. The low titer is potentially caused by 


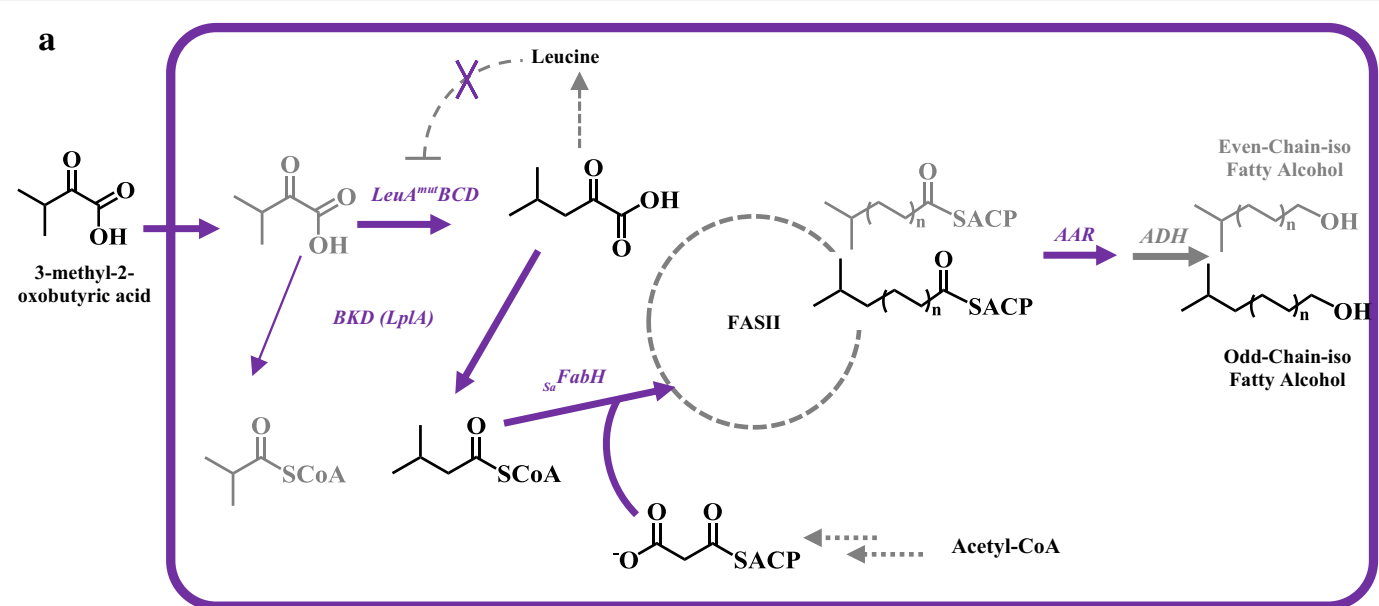

b
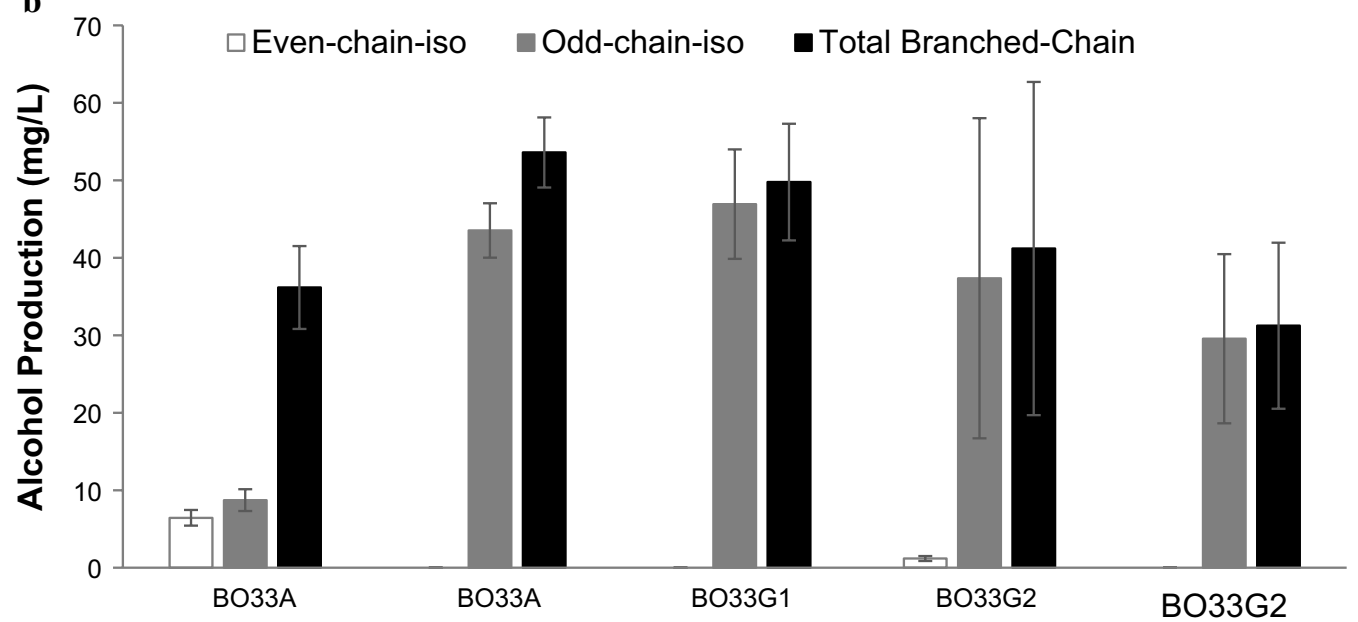

\begin{tabular}{|c|c|c|c|c|c|}
\hline $\operatorname{leu} A^{\mathrm{mut}} B C D$ & -- & -- & pA15a::Р & pColE1::Р & pColE1::PBAD \\
\hline $\begin{array}{l}\text { Inducer for } \\
\operatorname{leu} A^{\mathrm{mut}} B C D\end{array}$ & -- & -- & $0.4 \%$ arabinose & $0.1 \%$ arabinose & $0.4 \%$ arabinose \\
\hline$\alpha$-keto acid & -- & $\begin{array}{c}\text { 4-methyl-2- } \\
\text { oxopentanoic acid }\end{array}$ & $\begin{array}{l}\text { 3-methyl-2- } \\
\text { oxobutyric acid }\end{array}$ & $\begin{array}{l}\text { 3-methyl-2- } \\
\text { oxobutyric acid }\end{array}$ & $\begin{array}{l}\text { 3-methyl-2- } \\
\text { oxobutyric acid }\end{array}$ \\
\hline
\end{tabular}

Fig. 4 Optimizing the 4-methyl-2-oxopentanoic acid generation module to produce odd-chain-iso fatty alcohols. a Biosynthetic pathways for the production of odd-chain-iso fatty alcohols. b Titers of engineered odd-chain-iso BLFL-producing strains. Strains BO33G1-2 containing leuA ${ }^{\text {mut }} B C D$ in plasmids with different copy numbers were cultivated as described in "Methods", and supplemented with $1 \mathrm{~g} / \mathrm{L}$ 3-methyl-2-oxobutyric acid. Strain BO33G2 was induced with either 0.1 or $0.4 \%$ arabinose

the insolubility of highly overexpressed AAR [13]. Meanwhile, compared to strain BO33F3 with the low-copy als $S$-ilvCD expression, strain BO33F with the mediumcopy alsS-ilvCD expression improved BLFL production by 3.2-fold. These results suggest that the optimal aar expression level was medium to low, and that alsS-ilv $C D$ required overexpression in a medium to high-copy number plasmid for optimal BLFL production (Fig. 3b; Additional file 1: Figure S1).

\section{BLFL production from glucose using the acyl-ACP reductase Maqu2220}

During the course of this work, Haushalter et al. published the production of straight LCFLs in high titers by overexpression of a marine acyl-ACP reductase, Maqu2220 from M. aquaeolei VT8 [48]. Maqu2220 was first characterized as a fatty aldehyde reductase, reducing fatty aldehydes to fatty alcohols [49]. However, it was later confirmed to have acyl-ACP reductase activity and 
is capable of converting acyl-ACPs or acyl-CoAs directly to fatty alcohols [50], thus significantly simplifying the fatty alcohol pathway. We tested the efficiency of BLFL production via Maqu2220 by replacing the S. elongatus aar in strain BO33A with maqu2220, resulting in strain BO33D. When supplemented with $1 \mathrm{~g} / \mathrm{L}$ of 4-methyl2-oxopentanoic acid, BO33D produced $198 \mathrm{mg} / \mathrm{L}$ (89\%) BC alcohols, 3.7-fold higher than that of strain BO33A (Fig. 5). Even compared with the previous best-performing strain BO33E3, the BLFL titer of strain BO33D enhanced by 1.5 -fold. Most BLFLs (> 99\%) were identified in the cell pellet, consistent with previous study [51]. Moreover, overexpression of the ADHs $y j g B, y q h D$, or adhA did not enhance BLFL titer (Fig. 5), further confirming the previous hypothesis that Maqu2220 contains a catalytic domain with $\mathrm{ADH}$ activity.

After confirming the capability of Maqu2220 to convert branched-chain acyl-ACPs to BLFLs, we sought to use Maqu2220 to produce BLFLs from glucose by applying the knowledge learned from optimizing the $\alpha$-keto acid synthesis module. After constructing and validating strains, we found that the optimal expression level of each module mimicked those characterized using the aar pathway. First, the high-copy number-expression (pColE1) of the alsS-ilvCD operon increased even-chainiso fatty alcohol production by 9.2 -fold over the lowcopy number-expression (pA15a) (as seen by comparing strains BO33I1 with BO33I2 in Fig. 6a). Second, when supplemented with 3-methyl-2-oxobutyric acid, the low-copy number-expression of the $l e u A^{\text {mut }} B C D$ operon produced 2.8-fold more odd-chain-iso fatty alcohols than the medium-copy number-expression (as seen for strains OB33J1 and OB33J2 in Fig. 6a).

Next, we attempted to combine all three modules to produce BLFLs from glucose, which requires overexpression of 14 genes from 6 synthetic operons: $\mathrm{P}_{\text {tet }}$-alsS-ilvCD, $\mathrm{P}_{\mathrm{BAD}}-\mathrm{leu} A^{\mathrm{mut}} B C D$, $\mathrm{P}_{\text {lacUV5-bs }} l p d V_{-b s} b k d A A_{-b s} b k d A B{ }_{b s} b k d B, \quad \mathrm{P}_{\text {lacUV5-lplA, }}$ $\mathrm{P}_{\text {ecfabH-Sa }}$ fabH, and $\mathrm{P}_{\text {lacUv5 }}-$ maqu2220. To reduce the burden imposed by multiple plasmids while maintaining a sufficient enzyme expression level, we tried to integrate some operons into the genome. Based on the findings above, we first created strain $\mathrm{BC} 43$ with a single copy leu $A^{\mathrm{mut}} B C D$ from strain BC33. Using the Type II CRISPR-Cas9 system, we replaced the native promoters of the genomic leuABCD operon with the strong IPTGinducible $\mathrm{P}_{\text {lacUv5 }}$ promoter, and replaced the RBS of leuA (including all regulatory sites) with the previously-used strong synthetic RBS. Simultaneously, we replaced the leuA-coding gene with the feedback-resistant leuA ${ }^{\mathrm{mu}-}$ tant. However, when the resulting strain $\mathrm{BC} 43$ was transformed with corresponding plasmids for fermentation, even-chain-iso products predominated over odd-chainiso products (as seen for strains BO43I in Fig. 6a). This result indicated that the $\operatorname{le} u A^{\text {mut }} B C D$ requires very fine-tuning and that a single copy of leu $A^{\text {mut }} B C D$ was insufficient to convert all the intracellular 3-methyl-2-oxobutyric acid to 4-methyl-2-oxopentanoic acid, further proving that $\mathrm{pA} 15 \mathrm{a}:: \mathrm{P}_{\mathrm{BAD}}-\mathrm{leu} A^{\mathrm{mut}} B C D$ provides optimal expression level for the $\alpha$-keto acid synthesis module.

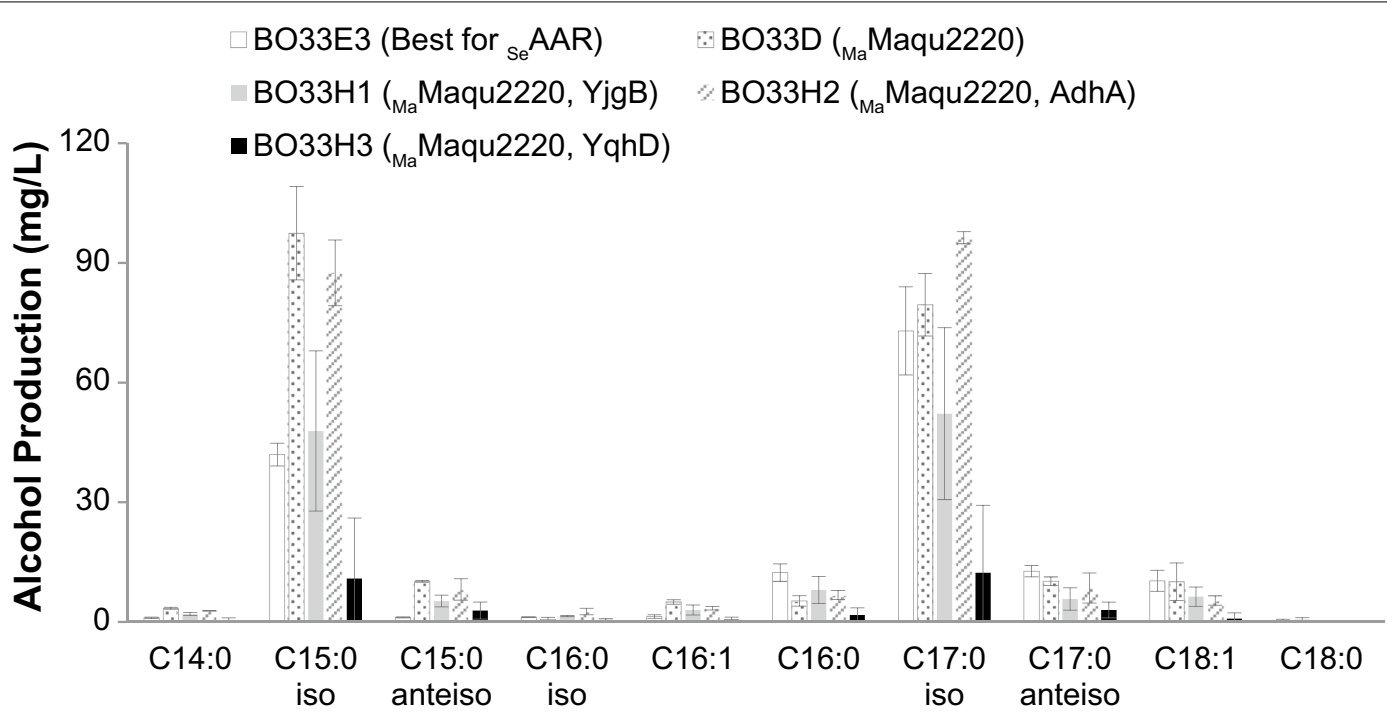

Fig. 5 BLFL profiles of engineered E. coli strains containing Maqu2220. While strain BO33D does not contain additional adh, BO33H1-3 overexpressed L. lactis adhA, E. coli yqhD, and E. coli yjgB, respectively. BLFL profiles are compared with that of strains BO33E3 (the best performing strain in "Engineering alcohol formation modules in BCFA-producing strains" section). All cultures were supplemented with $1 \mathrm{~g} / \mathrm{L}$ 4-methyl-2-oxopentanoic acid 
a $\square$ Even-Chain-iso $\quad$ Odd-Chain-iso $\quad \mathbf{T}$ Total Branched-Chain

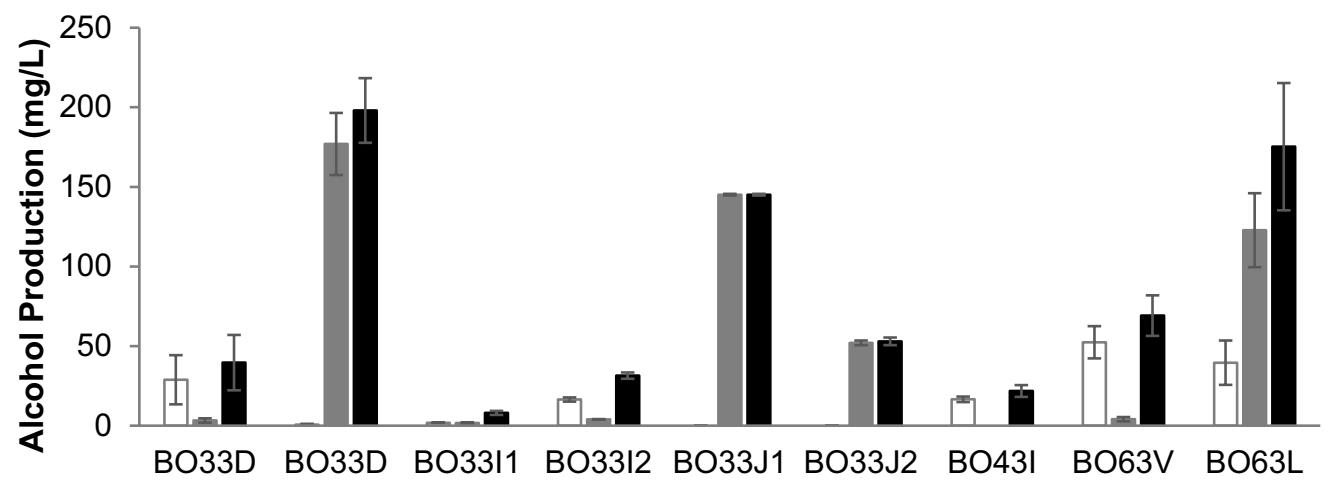

\begin{tabular}{|c|c|c|c|c|c|c|c|c|c|}
\hline$\alpha$-keto acid & $\begin{array}{l}\text { 3-methyl-2- } \\
\text { oxobutyric } \\
\text { acid }\end{array}$ & $\begin{array}{l}\text { 4-methyl-2- } \\
\text { oxopentanoic } \\
\text { acid }\end{array}$ & -- & -- & $\begin{array}{l}\text { 3-methyl-2- } \\
\text { oxobutyric } \\
\text { acid }\end{array}$ & $\begin{array}{c}\text { 3-methyl-2- } \\
\text { oxobutyric } \\
\text { acid }\end{array}$ & -- & -- & -- \\
\hline alsS-ilvCD & -- & -- & pA15a & $\mathrm{pCoE} 1$ & -- & -- & pCoE1 & pCoE1 & pCoE1 \\
\hline $\operatorname{leu} A^{\mathrm{mut}} B C D$ & -- & -- & -- & -- & $\mathrm{pA} 15 \mathrm{a}$ & pBBR1 & genome & -- & pA15a \\
\hline lplA & pBBR1 & pBBR1 & pBBR1 & pBBR1 & pBBR1 & pA15a & pBBR1 & genome & genome \\
\hline fadR & -- & -- & -- & -- & -- & -- & -. & genome & genome \\
\hline
\end{tabular}

b Glucose

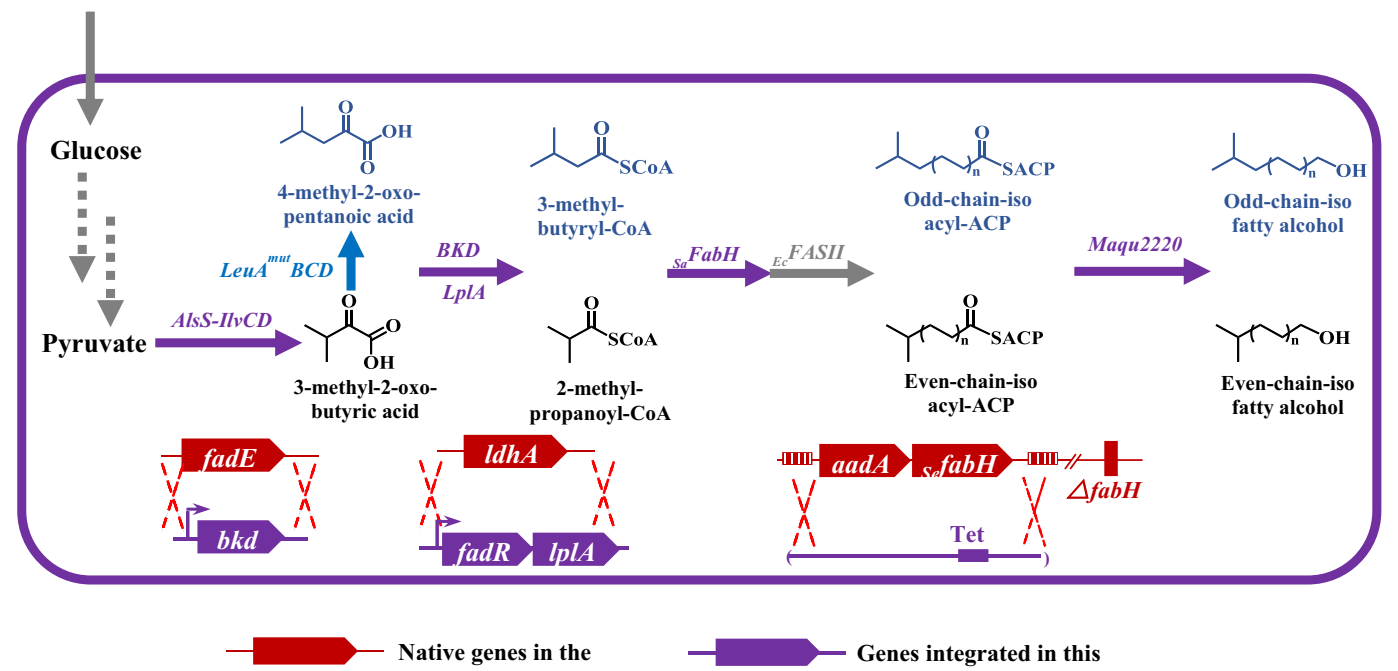

Fig. 6 Optimizing a-keto acid biosynthetic pathways in alcohol-producing strains containing ${ }_{\mathrm{Ma}}$ Maqu2220. a Plasmids with different copy numbers and promoters were employed to bear the involved genes in different strains. The titers of even-chain-iso, odd-chain-iso, and total alcohol from all the strains containing a-keto acid biosynthetic pathways are compared with that of strain BO33D with either $1 \mathrm{~g} / \mathrm{L}$ 3-methyl-2-oxobutyric acid or $1 \mathrm{~g} / \mathrm{L}$ 4-methyl-2-oxopentanoic acid supplementation. b The complete pathways and genome modifications involved in strain BC63V (purple) and BC63L (purple and blue)

Consistency in expression parameters indicates that the optimal levels derived in this work may be applicable in other systems requiring the use of an $\alpha$-keto acid pathway.

Then, we chose lplA as the gene to be integrated into the host cell's genome; low expression was previously determined to be sufficient to fully functionalize the BKD
E2 subunit [27]. Furthermore, it has been demonstrated that even low-level overexpression of the global regulator of fatty acid metabolism $f a d R$ increases fatty acid production $[52,53]$. Thus, $f a d R$ was cloned to the same operon of lplA under the control of the $\mathrm{P}_{\mathrm{LacUv} 5}$ promoter. The synthetic $\mathrm{P}_{\mathrm{LacUv5}}-f a d R$-lplA operon was then integrated into the native $l d h A$ (involved in lactate production) site 
of the strain $\mathrm{BC} 43$, resulting in strain BC63 (Fig. 6b). Deletion of $l d h A$ was expected to reduce lactate formation during fermentation [54].

Strain BC63 was then engineered to produce BLFL with controlled chain structure. To produce even-chainiso BLFLs from glucose, we created strain BO63V by co-transforming the plasmids pB5k-maqu2220 and pE2salsS-ilvCD into strain BC63 (Table 2, Fig. 6b). When fermented in the absence of $\alpha$-keto acid, BO63V produced $52.4 \mathrm{mg} / \mathrm{L}$ of even-chain-iso fatty alcohols (Fig. 6a; Additional file 1: Figure S4). To produce odd-chain-iso fatty alcohols from glucose, the plasmid pA8c-leuA ${ }^{\text {mut }} B C D$ was further transformed to strain BO63 V (Fig. 6b). The resulting strain BO63L produced $122 \mathrm{mg} / \mathrm{L}$ of odd-chainiso BLFLs, comprising $70 \%$ of the total BLFLs (Fig. 6a; Additional file 1: Figure S4). Although the odd-chainiso BLFL pathway is less carbon efficient than the evenchain-iso BLFL pathway due to the loss of a $\mathrm{CO}_{2}$ during 4-methyl-2-oxopentanoic acid biosynthesis, the ${ }_{\mathrm{Sa}} \mathrm{FabH}$ has higher specificity towards 3-methylbutyryl-CoA (the precursor for odd-chain-iso products) than 2-methylpropanoyl-CoA (the precursor for even-chain-iso products) [28], thus leading to higher odd-chain-iso BLFL titer.

Finally, we assessed the performance of strain BO63L in a $1 \mathrm{~L}$ glucose fed-batch fermenter ("Fermentation" section). After $85 \mathrm{~h}$ of cultivation, BLFLs accumulated to $350 \mathrm{mg} / \mathrm{L}$ (Fig. 7a). The concentration of 14-methylpentadecanol, the most abundant product, reached $217 \mathrm{mg} / \mathrm{L}$ (Fig. 7b). Interestingly, straight LCFLs accumulated rapidly after induction, while BLFL titers did not increase until $32 \mathrm{~h}$ (Fig. 7a). We suspect that the initial straight-chain alcohol accumulation may result from the leaky expression of Maqu2220 prior to induction. Further optimization of fermentation conditions (such as $\mathrm{pH}$, air flowing rate, stirring rate, etc.) might improve the percentage and yield of BLFL. Overall, these results demonstrate the potential of strain BO63L for high-titer production of BLFLs and suggest that further studies might lead to its use on an industrial scale.

\section{Discussion}

Although alcohol-producing pathways have been previously engineered to produce straight LCFLs and shortchain alcohols, little effort has been made to produce BLFLs. In this work, we characterized the capability and substrate specificity of four different alcohol-producing pathways for BLFL production. We demonstrated the highly selective production of two types of BLFLs (odd-chain-iso and even-chain-iso) by engineering the upstream pathways for precursor synthesis. We obtained high BLFL proportions out of total fatty alcohols (strain BO63L, yielded 80\%). We also obtained comparable BLFL titers (strain BO63L, $175 \mathrm{mg} / \mathrm{L}$ in the absence of any precursor) to BCFA titers, despite the extensive additional engineering required for BLFLs production. The modular engineering strategy allowed us to apply the knowledge learned from the AAR pathway to the new identified Maqu2220 pathway for rapid optimization, quickly yielded strain BO63L that produced $350 \mathrm{mg} / \mathrm{L}$ BLFLs in a fed-batch fermenter. Because BLFLs can be directly used in skin-care or sunscreen products, and are good candidates for diesel fuels, our ability to produce specific BLFL species in high percentages may directly benefit the cosmetics and bioenergy fields.

Meanwhile, we partitioned the complete pathway into three modules: a precursor formation module (the $\alpha$-keto acid synthesis module), an acyl-CoA activation and malonyl-ACP consumption module (the acyl-ACP generation module), and a final product synthesis module (the alcohol formation module). Each module can be separately engineered and tuned for novel chemical synthesis. Furthermore, when the tuned modules are combined, the expression level of each module can be tuned to avoid imbalance and to improve product titer. We demonstrated consistent expression parameters that result in optimal productivities, which can be used for the production of other chemicals. Together with previous successes in modular engineering of the isoprenoid pathway [55], the FFA pathways [56] and the ester pathways [57], we confirm that modular pathway engineering is an effective approach to improve product titer and yield.

The top-performing strain BO63L produced $350 \mathrm{mg} / \mathrm{L}$ BLFLs in fed-batch fermenter, still low compared to that of the best straight LCFL-producing strain $(3.82 \mathrm{~g} / \mathrm{L}$ in shake flask) [48]. Our current pathway is not limited by $\alpha$-keto acid because increasing the supplemented 4-methyl-2-oxopentanoic acid from 1 to $8 \mathrm{~g} / \mathrm{L}$ did not increase BLFL titer (Additional file 1: Figure S3). Furthermore, conversion of acyl-ACPs to BLFLs is not likely to be the limiting step due to the high conversion efficiency of Maqu2220 [48]. Thus, we believe the current bottleneck lies in the acyl-ACP generation module, which requires further engineering to improve BLFL titer and yield. Previous engineering strategies developed to optimize free fatty acids production could be potentially used to further improve BLFL yield. These strategies include modulation of the fatty acid and the phospholipid biosynthesis pathway [58], implementing synthetic control systems to dynamically regulate pathway gene expression [52, 59-61], and enriching the high-performing subpopulation using PopQC [60]. 


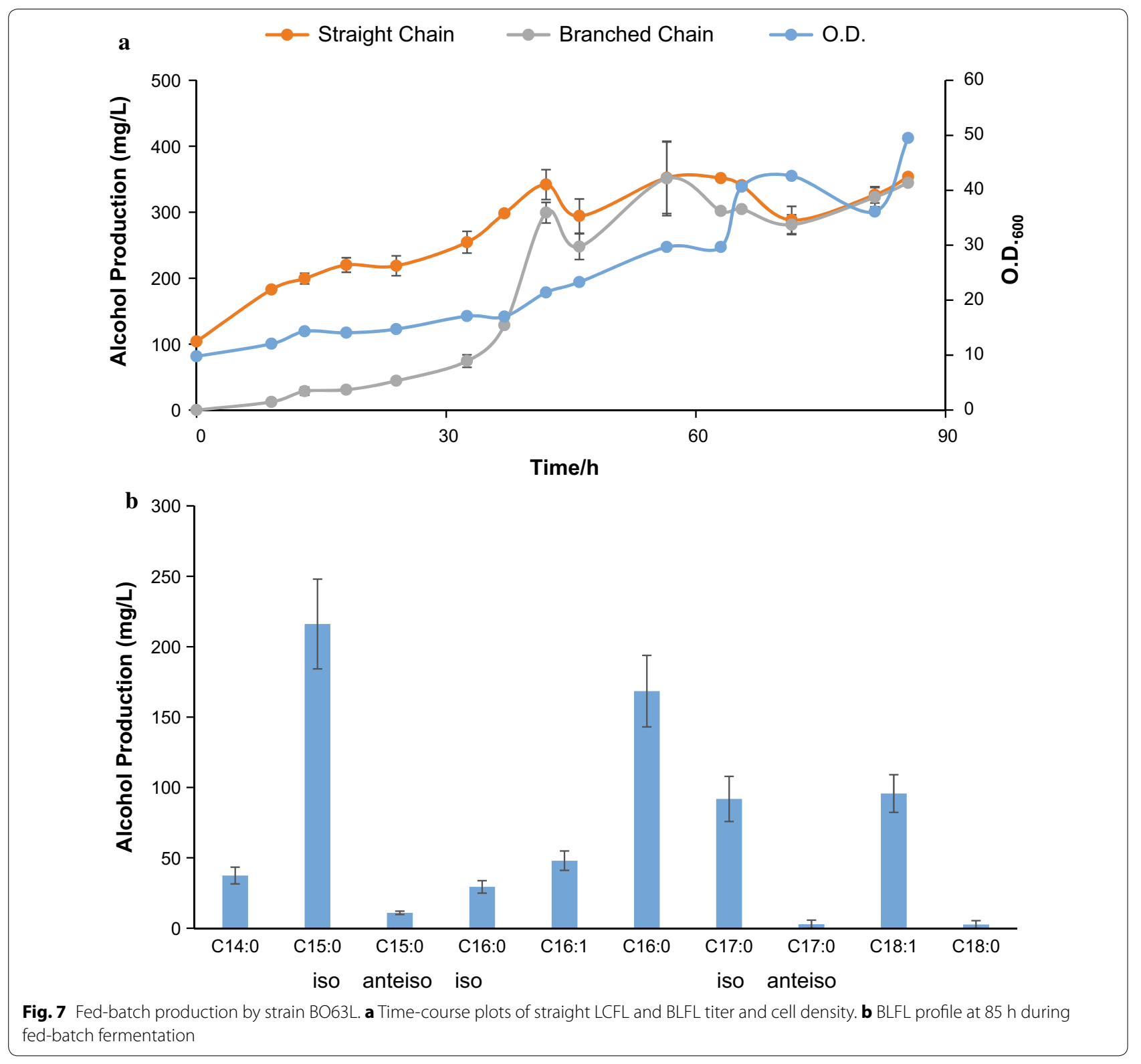

\section{Conclusions}

We have constructed and tested the capability of four different alcohol-producing pathways for BLFL production in engineered $E$. coli. Moreover, by engineering the $\alpha$-keto acid biosynthetic pathways and balancing the expression levels of three different modules, we achieved BLFL titers of up to $350 \mathrm{mg} / \mathrm{L}$ from glucose, and BLFL percentages up to $79 \%$. Overall, this work generates pathways and knowledge for the production of BLFLs in high percentages that will have broader industrial applications than straight LCFLs.

\section{Additional file}

Additional file 1: Figure S1. Fatty alcohol profiles of strains involved in Fig. 3b. Figure S2. Fatty alcohol profiles of strains involved in Fig. 4b. Figure S3. Odd-chain-iso fatty alcohol production in strain BO33D with different concentrations of 4-methyl-2-oxopentanoic acid supplementation. The data has been normalized to the odd-chain-iso fatty alcohol production in strain BO33D with $1 \mathrm{~g} / \mathrm{L}$ 4-methyl-2-oxopentanoic acid supplementation. Figure S4. Fatty alcohol profiles of strains involved in Fig. 6a. 


\section{Abbreviations}

BLFL: branched-long-chain fatty alcohol; ACP: acyl carrier protein; AAR: acyl-ACP reductase; CAR: carboxylic acid reductase; ACR: acyl-CoA reductase; FFA: free fatty acid; ADH: alcohol dehydrogenase; LCFL: long-chain fatty alcohol; BCFAs: branched-chain fatty acids; BKD: branched-chain a-keto acid dehydrogenase complex; FabH: $\beta$-ketoacyl-acyl-carrier protein; IPTG: $\beta$-D-1thiogalactopyranoside; aTc: anhydrotetracycline; BSTFA: N,O-bis(trimethylsilyl) trifluoroacetamide

\section{Authors' contributions}

WJ and FZ designed the work. WJ and JQ performed the plasmids and strain constructions, cell culture, fatty alcohol extraction and analysis. WJ and DL performed the fermenter experiment. WJ, GB and FZ wrote the manuscript. All authors read and approved the final manuscript.

\section{Author details}

${ }^{1}$ Department of Energy, Environmental and Chemical Engineering, Washington University in St. Louis, 1 Brookings Drive, Campus Box 1180, Saint Louis, MO 63130, USA. ${ }^{2}$ Division of Biological \& Biomedical Sciences, Washington University in St. Louis, Saint Louis, MO 63130, USA. ${ }^{3}$ Institute of Materials Science \& Engineering, Washington University in St. Louis, Saint Louis, MO 63130, USA. ${ }^{4}$ Present Address: National Bioenergy Center, National Renewable Energy Laboratory, Golden, CO 80401, USA.

\section{Acknowledgements}

The authors thank Dr. John Cronan for providing the parent strain CL111, and thank Dr. Brett Barney and Joint Bioenergy Institute to provide plasmids containing maqu2507 and maqu2220.

\section{Competing interests}

WJ, GB and FZ filed a patent entitled "Host cells and methods for producing fatty acid-derivatives with high branched-chain percentage", which is supplemented by this contribution.

\section{Availability of data and materials}

All data generated or analysed during this study are included in this published article and its additional files.

\section{Consent for publication}

Not applicable.

\section{Ethics approval and consent to participate}

Not applicable.

\section{Funding}

This work was supported by the Defense Advanced Research Projects Agency (D13AP00038) and the Natural Science Foundation (MCB1453147).

\section{Publisher's Note}

Springer Nature remains neutral with regard to jurisdictional claims in published maps and institutional affiliations.

Received: 9 July 2017 Accepted: 19 October 2017

Published online: 27 October 2017

\section{References}

1. Peralta-Yahya PP, Zhang F, del Cardayre SB, Keasling JD. Microbial engineering for the production of advanced biofuels. Nature. 2012;488:320-8.

2. Yadav VG, De Mey M, Giaw Lim C, Kumaran Ajikumar P, Stephanopoulos $G$. The future of metabolic engineering and synthetic biology: towards a systematic practice. Metab Eng. 2012;14:233-41.

3. Garg S, Rizhsky L, Jin HN, Yu XC, Jing FY, Yandeau-Nelson MD, Nikolau BJ. Microbial production of bi-functional molecules by diversification of the fatty acid pathway. Metab Eng. 2016;35:9-20.

4. Zargar A, Bailey CB, Haushalter RW, Eiben CB, Katz L, Keasling JD. Leveraging microbial biosynthetic pathways for the generation of 'drop-in' biofuels. Curr Opin Biotechnol. 2017;45:156-63.
5. Akhtar MK, Turner NJ, Jones PR. Carboxylic acid reductase is a versatile enzyme for the conversion of fatty acids into fuels and chemical commodities. Proc Natl Acad Sci USA. 2013;110:87-92.

6. Knaut J, Richtler HJ. Trends in industrial uses of palm and lauric oils. J Am Oil Chem Soc. 1985:62:317-27.

7. Carroll AL, Desai SH, Atsumi S. Microbial production of scent and flavor compounds. Curr Opin Biotechnol. 2016:37:8-15.

8. Washecheck PH. Manufacture of higher straight-chain alcohols by the ethylene chain growth process. In: Monohydric alcohols. vol. 159, : ACS Symposium Series. Washington: American Chemical Society; 1981. p. 87-100.

9. Voeste T, Buchold $\mathrm{H}$. Production of fatty alcohols from fatty-acids. J Am Oil Chem Soc. 1984;61:350-2.

10. Youngquist JT, Schumacher MH, Rose JP, Raines TC, Politz MC, Copeland MF, Pfleger BF. Production of medium chain length fatty alcohols from glucose in Escherichia coli. Metab Eng. 2013;20:177-86.

11. Zheng YN, Li LL, Liu Q, Yang JM, Wang XW, Liu W, Xu X, Liu H, Zhao G, Xian M. Optimization of fatty alcohol biosynthesis pathway for selectively enhanced production of C12/14 and C16/18 fatty alcohols in engineered Escherichia coli. Microb Cell Fact. 2012:11:65.

12. Liu A, Tan X, Yao L, Lu X. Fatty alcohol production in engineered E. coli expressing Marinobacter fatty acyl-CoA reductases. Appl Microbiol Biotechnol. 2013:97:7061-71.

13. Liu R, Zhu FY, Lu L, Fu AS, Lu JK, Deng ZX, Liu TG. Metabolic engineering of fatty acyl-ACP reductase-dependent pathway to improve fatty alcohol production in Escherichia coli. Metab Eng. 2014;22:10-21.

14. Sheng JY, Stevens J, Feng XY. Pathway compartmentalization in peroxisome of Saccharomyces cerevisiae to produce versatile medium chain fatty alcohols. Sci Rep. 2016:6:26884.

15. Sheng JY, Feng XY. Metabolic engineering of yeast to produce fatty acidderived biofuels: bottlenecks and solutions. Front Microbiol. 2015;6:554.

16. Gabriels D, Hernandez WY, Sels B, Van Der Voort P, Verberckmoes A. Review of catalytic systems and thermodynamics for the Guerbet condensation reaction and challenges for biomass valorization. Catal Sci Technol. 2015;5:3876-902.

17. O'Lenick AJ. Guerbet chemistry. J Surfactants Deterg. 2001;4:311-5.

18. Mueller G, Bongardt F, Fies M, Daute P. Hydraulic oils containing biodegradable guerbet alcohols. Google Patents; 1996

19. Vinson PK, Foley PR, Cripe TA, Connor DS, Willman KW. Detergent compositions containing selected mid-chain branched surfactants. Google Patents; 2001

20. Dyer DJ, Walba DM. Improvement of thermotropic liquid crystallinity by incorporation of unsaturated fatty alcohol tail units. Chem Mater. 1994;6:1096-8

21. Kozlowski JT, Davis RJ. Heterogeneous catalysts for the guerbet coupling of alcohols. Acs Catal. 2013:3:1588-600.

22. Anbarasan P, Baer ZC, Sreekumar S, Gross E, Binder JB, Blanch HW, Clark DS, Toste FD. Integration of chemical catalysis with extractive fermentation to produce fuels. Nature. 2012:491:235-9.

23. Carlini C, Macinai A, Galletti AMR, Sbrana G. Selective synthesis of 2-ethyl1-hexanol from $n$-butanol through the Guerbet reaction by using bifunctional catalysts based on copper or palladium precursors and sodium butoxide. J Mol Catal Chem. 2004:212:65-70.

24. Marcheschi RJ, Li H, Zhang KC, Noey EL, Kim S, Chaubey A, Houk KN, Liao JC. A Synthetic recursive "+1" pathway for carbon chain elongation. ACS Chem Biol. 2012;7:689-97

25. Sheppard MJ, Kunjapur AM, Wenck SJ, Prather KLJ. Retro-biosynthetic screening of a modular pathway design achieves selective route for microbial synthesis of 4-methyl-pentanol. Nat Commun. 2014;5:5031.

26. Lennen RM, Pfleger BF. Microbial production of fatty acid-derived fuels and chemicals. Curr Opin Biotechnol. 2013;24:1044-53.

27. Bentley GJ, Jiang W, Guaman LP, Xiao Y, Zhang F. Engineering Escherichia coli to produce branched-chain fatty acids in high percentages. Metab Eng. 2016:38:148-58.

28. Jiang W, Jiang Y, Bentley GJ, Liu D, Xiao Y, Zhang F. Enhanced production of branched-chain fatty acids by replacing-ketoacyl-(acyl-carrier-protein) synthase III (FabH). Biotechnol Bioeng. 2015;112:1613-22.

29. McMahon MD, Prather KLJ. Functional screening and in vitro analysis reveal thioesterases with enhanced substrate specificity profiles that improve short-chain fatty acid production in Escherichia coli. Appl Environ Microbiol. 2014;80:1042-50. 
30. Martin CH, Dhamankar H, Tseng HC, Sheppard MJ, Reisch CR, Prather KLJ. A platform pathway for production of 3-hydroxyacids provides a biosynthetic route to 3-hydroxy-gamma-butyrolactone. Nat Commun. 2013:4:1414.

31. Jones JA, Toparlak OD, Koffas MAG. Metabolic pathway balancing and its role in the production of biofuels and chemicals. Curr Opin Biotechnol. 2015;33:52-9

32. Zhao SJ, Jones JA, Lachance DM, Bhan N, Khalidi O, Venkataraman S, Wang ZT, Koffas MAG. Improvement of catechin production in Escherichia coli through combinatorial metabolic engineering. Metab Eng. 2015:28:43-53.

33. Jones JA, Vernacchio VR, Lachance DM, Lebovich M, Fu L, Shirke AN, Schultz VL, Cress B, Linhardt RJ, Koffas MAG. ePathOptimize: a combinatorial approach for transcriptional balancing of metabolic pathways. Sci Rep. 2015;5:11301.

34. Fang MY, Wang TM, Zhang C, Bai JL, Zheng X, Zhao XJ, Lou CB, Xing XH. Intermediate-sensor assisted push-pull strategy and its application in heterologous deoxyviolacein production in Escherichia coli. Metab Eng. 2016;33:41-51.

35. Lee TS, Krupa RA, Zhang F, Hajimorad M, Holtz WJ, Prasad N, Lee SK Keasling JD. BglBrick vectors and datasheets: a synthetic biology platform for gene expression. J Biol Eng. 2011;5:12.

36. Engler C, Kandzia R, Marillonnet S. A one pot, one step, precision cloning method with high throughput capability. PLoS ONE. 2008;3:e3647.

37. Lai CY, Cronan JE. beta-ketoacyl-acyl carrier protein synthase III $(\mathrm{FabH})$ is essential for bacterial fatty acid synthesis. J Biol Chem. 2003;278:51494-503.

38. Datsenko KA, Wanner BL. One-step inactivation of chromosomal genes in Escherichia coli K-12 using PCR products. Proc Natl Acad Sci USA. 2000;97:6640-5.

39. Jiang Y, Chen B, Duan CL, Sun BB, Yang JJ, Yang S. Multigene editing in the Escherichia coli genome via the CRISPR-Cas9 System. Appl Environ Microbiol. 2015;81:2506-14.

40. Willis RM, Wahlen BD, Seefeldt LC, Barney BM. Characterization of a fatty acyl-coa reductase from Marinobacter aquaeolei VT8: a bacterial enzyme catalyzing the reduction of fatty acyl-coa to fatty alcohol. Biochemistry. 2011;50:10550-8

41. Schirmer A, Rude MA, Li XZ, Popova E, del Cardayre SB. Microbial biosynthesis of alkanes. Science. 2010;329:559-62.

42. Steen EJ, Kang YS, Bokinsky G, Hu ZH, Schirmer A, McClure A, del Cardayre SB, Keasling JD. Microbial production of fatty-acid-derived fuels and chemicals from plant biomass. Nature. 2010:463:559.

43. Atsumi S, Wu TY, Eckl EM, Hawkins SD, Buelter T, Liao JC. Engineering the isobutanol biosynthetic pathway in Escherichia coli by comparison of three aldehyde reductase/alcohol dehydrogenase genes. Appl Microbiol Biotechnol. 2010;85:651-7.

44. Jarboe LR. YqhD: a broad-substrate range aldehyde reductase with various applications in production of biorenewable fuels and chemicals. Appl Microbiol Biotechnol. 2011;89:249-57.

45. Stieglitz BI, Calvo JM. Distribution of the isopropylmalate pathway to leucine among diverse bacteria. J Bacteriol. 1974;118:935-41.
46. Connor MR, Liao JC. Engineering of an Escherichia coli strain for the production of 3-methyl-1-butanol. Appl Environ Microbiol. 2008;74:5769-75.

47. Gusyatiner MM, Ivanovskaya LV, Kozlov YI, Lunts MG, Voroshilova EB. DNA coding for mutant isopropylmalate synthase, L-leucine-producing microorganism and method for producing L-leucine. Google Patents; 2005.

48. Haushalter RW, Groff D, Deutsch S, The L, Chavkin TA, Brunner SF, Katz L, Keasling JD. Development of an orthogonal fatty acid biosynthesis system in E. coli for oleochemical production. Metab Eng. 2015;30:1-6.

49. Wahlen BD, Oswald WS, Seefeldt LC, Barney BM. Purification, characterization, and potential bacterial wax production role of an NADPH-dependent fatty aldehyde reductase from Marinobacter aquaeoleiVT8. Appl Environ Microbiol. 2009;75:2758-64.

50. Hofvander P, Doan TTP, Hamberg M. A prokaryotic acyl-CoA reductase performing reduction of fatty acyl-CoA to fatty alcohol. FEBS Lett. 2011;585:3538-43.

51. Liu YL, Chen S, Chen JJ, Zhou JM, Wang YY, Yang MH, Qi XN, Xing JM, Wang QH, Ma YH. High production of fatty alcohols in Escherichia coli with fatty acid starvation. Microb Cell Fact. 2016:15:129.

52. Zhang F, Carothers JM, Keasling JD. Design of a dynamic sensor-regulator system for production of chemicals and fuels derived from fatty acids. Nat Biotechnol. 2012;30:354.

53. Zhang F, Ouellet M, Batth TS, Adams PD, Petzold CJ, Mukhopadhyay A Keasling JD. Enhancing fatty acid production by the expression of the regulatory transcription factor FadR. Metab Eng. 2012;14:653-60.

54. Atsumi S, Hanai T, Liao JC. Non-fermentative pathways for synthesis of branched-chain higher alcohols as biofuels. Nature. 2008;451:86.

55. Ajikumar PK, Xiao W-H, Tyo KEJ, Wang Y, Simeon F, Leonard E, Mucha O, Phon TH, Pfeifer B, Stephanopoulos G. Isoprenoid pathway optimization for taxol precursor overproduction in Escherichia coli. Science. 2010;330:70-4.

56. Xu P, Gu Q, Wang WY, Wong L, Bower AGW, Collins CH, Koffas MAG. Modular optimization of multi-gene pathways for fatty acids production in E. coli. Nat Commun. 2013:4:1409.

57. Layton DS, Trinh CT. Expanding the modular ester fermentative pathways for combinatorial biosynthesis of esters from volatile organic acids. Biotechnol Bioeng. 2016;113:1764-76.

58. Fatma Z, Jawed K, Mattam AJ, Yazdani SS. Identification of long chain specific aldehyde reductase and its use in enhanced fatty alcohol production in E. coli. Metab Eng. 2016;37:35-45.

59. Liu D, Xiao Y, Evans BS, Zhang F. Negative feedback regulation of fatty acid production based on a malonyl-CoA sensor-actuator. ACS Synth Biol. 2015:4:132-40.

60. Xiao Y, Bowen CH, Liu D, Zhang F. Exploiting nongenetic cell-to-cell variation for enhanced biosynthesis. Nat Chem Biol. 2016;12:339.

61. Xu P, Li LY, Zhang FM, Stephanopoulos G, Koffas M. Improving fatty acids production by engineering dynamic pathway regulation and metabolic control. Proc Natl Acad Sci USA. 2014;111:11299-304.

\section{Submit your next manuscript to BioMed Central and we will help you at every step:}

- We accept pre-submission inquiries

- Our selector tool helps you to find the most relevant journal

- We provide round the clock customer support

- Convenient online submission

- Thorough peer review

- Inclusion in PubMed and all major indexing services

- Maximum visibility for your research

Submit your manuscript at www.biomedcentral.com/submit
BioMed Central 